\title{
Balanced Steady-State Free Precision and Time of Flight Noncontrast Magnetic Resonance Angiography in Peripheral Arterial Disease
}

\author{
Soumya Susan Regi ${ }^{1} \quad$ Aparna Irodi $^{1}$ Shyamkumar N. Keshava ${ }^{2}$ Sunil Agarwal ${ }^{3}$ \\ ${ }^{1}$ Division of Clinical Radiology, Department of Diagnostic Radiology, \\ Christian Medical College, Vellore, Tamil Nadu, India \\ 2 Division of Clinical Radiology, Department of Interventional \\ Radiology, Christian Medical College, Vellore, Tamil Nadu, India

\begin{abstract}
Address for correspondence Aparna Irodi, MBBS, MD, FRCR, Division of Clinical Radiology, Department of Diagnostic Radiology, Christian Medical College Hospital, Vellore, Tamil Nadu 632004, India (e-mail: aparnashyam@gmail.com).
\end{abstract}

${ }^{3}$ Department of Vascular Surgery, Christian Medical College, Vellore,

Tamil Nadu, India

J Clin Interv Radiol ISVIR 2022;6:98-105.

Purpose To determine the diagnostic efficacy of balanced steady-state free precession (bSSFP) and time-of-flight (TOF)-based noncontrast magnetic resonance angiography (NC-MRA) in lower limb peripheral arterial disease (PAD).

Methods Ten patients with suspected PAD underwent both NC-MRA (bSSFP and 2D TOF) and contrast-enhanced MR angiography (CE-MRA)/CT angiography (CTA). A total of 170 arterial segments (17 segments in each patient) were analyzed on NC-MRA and compared with CE-MRA/CTA for quality of images and for estimating the degree of stenoses. Image quality was graded as 1-poor, 2-fair, 3-good, and 4-excellent. The degree of stenoses was graded as $0-$ normal, $1-<50 \%$ narrowing, $2->50 \%$ narrowing, 3-near complete/100\% occlusion. Sensitivity, specificity, positive predictive value, and negative predictive value of NC-MRA in identifying significant stenosis, as compared with CE-MRA/CTA, were estimated.

Results a) Mean grade of the image quality of NC-MRA was 3.10 and the CE-MRA/CTA was 3.64. b) The agreement in the estimation of the degree of stenosis on NC-MRA as compared with CE-MRA/CTA was substantial in aortoiliac segments (weighted kappa 0.646 [95\% Cl] [0.361-0.931] [ $<<0.001])$, almost perfect in femoropopliteal segments (weighted kappa 0.911 [95\% Cl] [0.79-1.032] [ $p<0.001]$ ), and poor in infrapopliteal segments (weighted kappa 0.052 [95\% Cl] [0.189-0.293] [ $<<0.33587]$ ). Conclusion TOF and bSSFP-based NC-MRA was found to be comparable to the CE-MRA/CTA in the evaluation of PAD in lower limbs in the aortoiliac and femoropopliteal regions. NC-MRA was especially helpful in assessing the aortoiliac vessels and femoropopliteal vessels, with the imaging of infrapopliteal arteries being suboptimal. published online July 6, 2021
DOI https://doi.org/ 10.1055/s-0041-1730845. ISSN 2457-0214.
(C) 2021. Indian Society of Vascular and Interventional Radiology. All rights reserved.

This is an open access article published by Thieme under the terms of the Creative Commons Attribution-NonDerivative-NonCommercial-License, permitting copying and reproduction so long as the original work is given appropriate credit. Contents may not be used for commercial purposes, or adapted, remixed, transformed or built upon. (https://creativecommons.org/ licenses/by-nc-nd/4.0/)

Thieme Medical and Scientific Publishers Pvt. Ltd., A-12, 2nd Floor, Sector 2, Noida-201301 UP, India 


\section{Introduction}

In clinical practice, $\mathrm{CT}$ angiography (CTA) has now replaced diagnostic digital subtraction angiography (DSA) in the evaluation of peripheral arterial disease (PAD). ${ }^{1-3}$ The risk of iodinated, contrast-related nephropathy and ionizing radiation are the drawbacks of CTA. ${ }^{4}$ Contrast-enhanced magnetic resonance angiography (CE-MRA) has been proven to have a high diagnostic accuracy in the assessment of PAD. ${ }^{4-7}$ However, adverse effects of gadolinium contrast, particularly nephrogenic systemic fibrosis (NSF) in patients with renal dysfunction, limits the use of CE-MRA in patients with renal dysfunction. ${ }^{8-11}$ In this study, we explore the efficacy of noncontrast magnetic resonance angiography (NC-MRA) as an alternative to CE-MRA.

Over the years, various NC-MRA techniques have been developed in the evaluation of PAD. Time-of-flight (TOF), one of the older techniques of NC-MRA, is a nonsubtractive inflowdependent technique. ${ }^{12}$ TOF acquisitions maybe by $2 \mathrm{D}$ or $3 \mathrm{D}$ sampling. Fast and robust gradient-echo (GRE) sequences like steady-state free precession (SSFP) angiography have overcome some of the pitfalls associated with the older techniques of NC-MRA. SSFP angiography uses a series of equidistant radiofrequency $(\mathrm{RF})$ pulses, which are applied in a steady-state of longitudinal and transverse magnetization. ${ }^{13,14}$ The image contrast is T2-/T1-weighted, ${ }^{15}$ in which high-signal intensity with little reliance on inflow is given to the blood.

This study was aimed at assessing the diagnostic performance of ECG triggered NC-MRA using bSSFP and 2D TOF in the evaluation of PAD in the lower limbs at a magnetic field strength of 1.5 Tesla $(T)$.

\section{Material and Methods}

The study was approved by our Institution Review Board. Adult patients, with suspected lower limb PAD, undergoing a
CE-MRA or CTA in our institution were included in the study and, after obtaining an informed consent, were subjected to an additional NC-MRA. Patients with metallic implants, arrhythmias, and patients with renal insufficiency (glomerular filtration rate [GFR] of less than $30 \mathrm{~mL} / \mathrm{min} / 1.73 \mathrm{~m}^{2}$ ) were excluded from the study. In eight patients, NC-MRA was performed, followed by CE-MRA for comparison. In the remaining two patients, who were unable to cooperate for CE-MRA despite multiple attempts, CTA was performed on a 64 slice CT machine (GE Discovery 750 HD). NC-MRA was compared with CTA in these two patients. DSA was not performed in any of the patients.

Relevant clinical profile and demographic data of the patients was obtained and analyzed.

NC-MRA: Sequences and Technique

NC-MRA was performed on a 1.5T MRI machine (Philips Intera Achieva1.5T MRI scanner). Balanced steady-state free precession (bSSFP) ( - Table 1) sequence was used for the aorta and iliac vessels. 2D TOF was used for acquiring images of the lower limbs. Multiple 2-dimensional inflow (M2DI) sequence ( - Table 1), a flow-compensated GRE ECG-gated 2D TOF sequence, was used for lower limb arteries. The patient was placed in the supine position and images were acquired in the axial plane from diaphragm to feet. Multiplanar, maximum intensity projection (MIP) and 3D reconstruction of the arteries were generated. Total acquisition time for NCMRA (bSSFP for aortoiliac region and 2D TOF for the femoropopliteal and infrapopliteal regions) was approximately 19 minutes ( $\mathbf{- T a b l e} \mathbf{1})$.

In patients who were in pain due to the significant PAD, oral analgesics (nonsteroidal anti-inflammatory drugs [NSAIDs]) were administered prior to the study.

CE-MRA Technique

CE-MRA was performed on a 1.5T MRI machine (Avanto, Siemens Systems, Germany). A 20 G intravenous (IV) cannula was inserted in the upper limb and the patient was placed in

Table 1 MRI parameters for CE-MRA and NC-MRA techniques

\begin{tabular}{|l|l|l|l|}
\hline Parameter & Contrast-enhanced MRA & Noncontrast MRA: TOF & Noncontrast MRA: BFFE (bSSFP) \\
\hline Coils used & $\begin{array}{l}\text { PA (peripheral matrix or boot), } \\
\text { and two body }\end{array}$ & Q body & Q body/torso coil \\
\hline Flip angle $\left(^{\circ}\right)$ & 25 & 65 & 90 \\
\hline FOV (mm) & 400 & 400 & 400 \\
\hline Matrix & $208 \times 256$ & $256 \times 128$ & $336 \times 256$ \\
\hline Cardiac synchronization & & ECG gated & ECG gated \\
\hline Acquisition time & $\begin{array}{l}\sim 4 \text { minutes } \\
(\text { for the entire lower limb) }\end{array}$ & $\sim 16$ minutes & $\begin{array}{l}\sim ~ 3 \text { minutes (for the aortoiliac } \\
\text { region alone) }\end{array}$ \\
\hline TR (ms) & 3.0 & 26 & 3.3 \\
\hline TE (ms) & 1.17 & 7.5 & 1.7 \\
\hline Bandwidth (Hz/pixel) & 888 & 183 & 961 \\
\hline $\begin{array}{l}\text { Plane of acquisition/ } \\
\text { slice orientation }\end{array}$ & Coronal & Axial & Axial \\
\hline Slice thickness & $6 \mathrm{~mm}$ & $5 \mathrm{~mm}$ & $4 \mathrm{~mm}$ \\
\hline
\end{tabular}

Abbreviations: BBFE, balanced fast-field echo; bSSFP, balanced steady-state free precession; CE-MRA, contrast-enhanced MR angiography; FOV, field of view; NC-MRA, noncontrast MR angiography; TOF, time of flight. 
the supine position with feet first in the gantry. Following localizers, 15 to $30 \mathrm{~mL}$ of gadolinium (gadoteric acid) was injected using the pressure injector at a rate of $2 \mathrm{~mL} / \mathrm{sec}$, followed by $20 \mathrm{~mL}$ of saline flush. Using the bolus tracking technique, the trigger being placed in the aorta, dynamic postcontrast images were obtained in a coronal plane from the diaphragm to the feet. Images were reconstructed and analyzed in different planes (axial and coronal). MIP and volume-rendered 3D reconstructions were obtained.

\section{Image Analysis}

The arteries were divided into the following 17 segments: aorta, right and left common iliac arteries (CIA), right and left external iliac arteries (EIA), right and left common femoral arteries (CFA), right and left superficial femoral arteries (SFA), right and left popliteal arteries, right and left anterior tibial arteries (ATA), right and left common peroneal arteries (CPA), and right and left posterior tibial arteries (PTA). In all, 170 arterial segments were analyzed in all the 10 patients, that is, 17 arterial segments in each patient.

Each of the segments was then evaluated and graded for the quality of the image and the degree of stenosis by two radiologists independently, who were blinded to subject identity and the overall diagnosis.

The arteries of the lower limbs were divided into the following three regions:

Region 1-aortoiliac-abdominal aorta, CIA and EIA; Region 2-femoropopliteal, CFA, SFA and popliteal arteries; Region 3infrapopliteal, arteries in the leg, CPA, ATA and PTA.

The quality of the images for each arterial segment was qualitatively graded as follows: 1-Poor; 2-Fair; 3-Good; 4 -Excellent.

The degree of stenosis was graded as follows: 0-no occlusion/normal; 1 -less than or equal to $50 \%$ narrowing of the vessel; $2-$ more than $50 \%$ narrowing of the vessel; $3-$ near complete/100\% occlusion of the vessel (-Fig. 1 and -Fig. 2). In arteries with more than one stenosis, the highest degree of stenosis was considered.

Segments which were not visualized in their entirety (either due to the artery completely being occluded or due
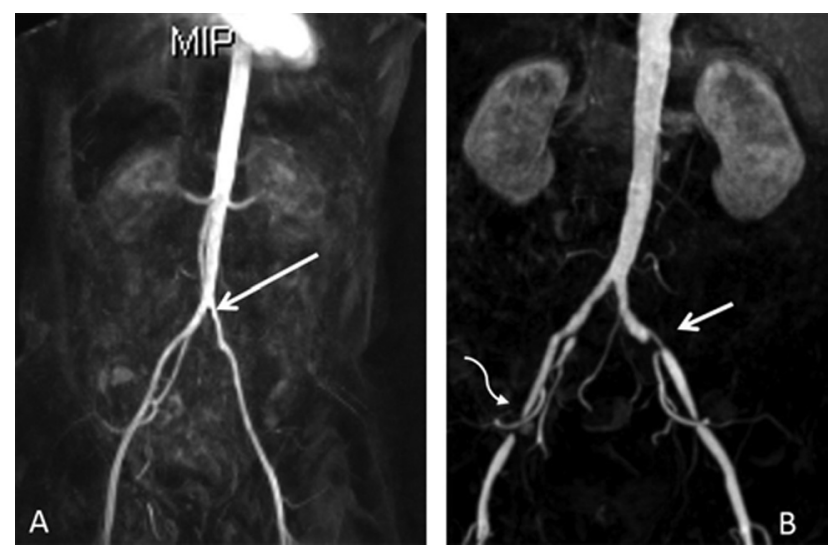

Fig. 1 Contrast-enhanced MR angiography (CE-MRA) in two different patients depicting the different grades of stenosis: (A) CE-MRA: grade 1 stenosis in the left common iliac artery (white arrow). (B) CE-MRA: grade 2 stenosis in the left common iliac artery (white arrow), with grade 3 stenosis in the right external iliac artery (curved white arrow).
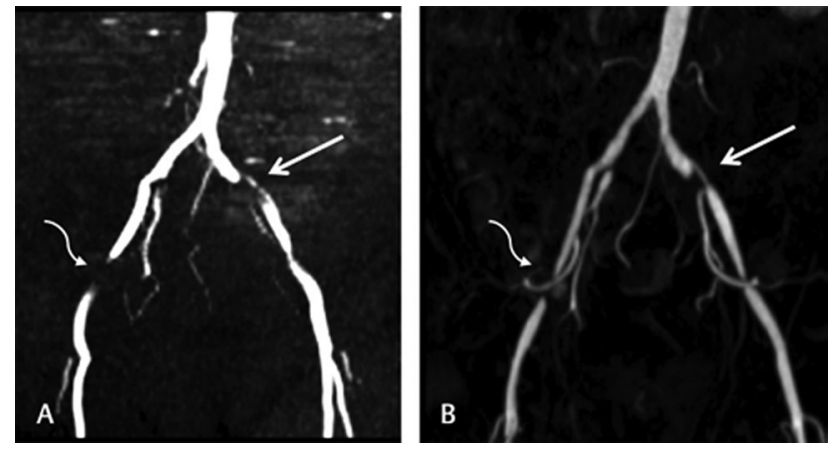

Fig. 2 Noncontrast MR angiography (NC-MRA) and CE-MRA in the same patient in the aortoiliac region. (A) NC-MRA: grade 3 stenosis (occlusion) of the right external iliac artery (curved white arrow) and grade 2 stenosis of the left external iliac artery. (B) CE-MRA: grade 3 stenosis (occlusion) of the right external iliac artery (curved white arrow) and grade 2 stenosis of the left common iliac artery (white arrow).

to the quality of the image) were not included only in the grading of the quality of the image, as NC-MRA and CE-M$\mathrm{RA} / \mathrm{CTA}$ are interpreted separately in this blinded study. One artery in the femoropopliteal region and two arteries in the infrapopliteal region were not included in the grading of quality in the NC-MRA. One artery in the femoropopliteal region was not included in the CE-MRA/CTA. However, in the assessment of the degree of stenosis, these segments have been included and graded as grade $3-$ near complete/100\% occlusion of the vessel.

Statistical Analysis

All statistical analysis was performed using the SPSS software (version 23.0, IBM).

The quality of the images was compared using the Wilcoxon signed rank test. The scoring of the quality of the images by the senior radiologist was considered for analysis.

Sensitivity, specificity, positive predictive value and negative predictive value of NC-MRA for detecting stenosis greater than 50\%, as compared with the CE-MRA/CTA (the reference standards), were calculated. Each of the arterial regions was then analyzed using Cohen's weighted kappa for evaluating the efficacy of NC-MRA. ${ }^{16,17}$

Weighted kappa was also used to assess the interobserver agreement to determine the reliability of the test.

\section{Results}

Ten consecutive patients were included in the study (all men; mean age, 54.4 years; age range, 34-66 years; mean pack years of smoking, 31 years; ankle brachial pressure index [ABPI]: a mean of 0.744 on the right [range $0.14-1.20$ ] and a mean of 0.632 on the left [range $0.00-1.10$ ]). Eight patients had diabetes mellitus and six patients were found to be hypertensive.

Image Quality

Image quality was assessed based on the senior radiologist's readings; hence, a total of 170 arterial segments were analyzed. The median score of the quality in NC-MRA was 4, which was the same as CE-MRA/CTA. The mean for the NC- 
Table 2 Mean scores of image quality of CE-MRA/CTA and NC-MRA

\begin{tabular}{|c|c|c|c|}
\hline Artery & CE-MRA/CTA & NC-MRA & $p$ value \\
\hline Aorta & $4 \pm 0$ & $3.8 \pm 0.42$ & 0.083 \\
\hline Common iliac artery & $3.95 \pm 0.22$ & $3.35 \pm 0.67$ & 0.003 \\
\hline External iliac artery & $3.95 \pm 0.22$ & $3.5 \pm 0.69$ & 0.021 \\
\hline Common femoral artery & $3.9 \pm 0.31$ & $3.9 \pm 0.31$ & 1.00 \\
\hline Superficial femoral artery & $3.75 \pm 0.44$ & $3.9 \pm 0.31$ & 0.180 \\
\hline Popliteal artery & $3.65 \pm 0.93$ & $3.75 \pm 0.91$ & 0.317 \\
\hline Anterior tibial artery & $3.25 \pm 1.12$ & $1.9 \pm 1.02$ & 0.001 \\
\hline Posterior tibial artery & $3.2 \pm 1.11$ & $1.9 \pm 1.08$ & 0.010 \\
\hline Common peroneal artery & $3.15 \pm 1.09$ & & \\
\hline
\end{tabular}

Abbreviations: CE-MRA, contrast-enhanced MR angiography; CTA, CT angiography; NC-MRA, noncontrast MR angiography.

Table 3 Scoring of the quality of images region-wise

\begin{tabular}{|c|c|c|c|c|c|}
\hline \multirow[t]{2}{*}{ Arterial region } & \multirow[t]{2}{*}{ Type of angiogram } & \multicolumn{4}{|c|}{ Grading } \\
\hline & & Excellent & Good & Fair & Poor \\
\hline \multirow[t]{2}{*}{ Aortoiliac $^{a}$} & CE-MRA/CTA & $48 / 50$ (96\%) & $2 / 50(4 \%)$ & $0(0 \%)$ & $0(0 \%)$ \\
\hline & NC-MRA & $29 / 50$ (58\%) & $17 / 50(34 \%)$ & $4 / 50(8 \%)$ & $0(0 \%)$ \\
\hline \multirow[t]{2}{*}{ Femoropopliteal $^{\text {b }}$} & CE-MRA/CTA & $\begin{array}{l}49 / 59 \\
(83 \%)\end{array}$ & $10 / 59$ (17\%) & $0(0 \%)$ & $0(0 \%)$ \\
\hline & NC-MRA & $54 / 59$ (92\%) & $5 / 59(8 \%)$ & $0(0 \%)$ & $0(0 \%)$ \\
\hline \multirow[t]{2}{*}{ Infrapopliteal c } & CE-MRA/CTA & $33 / 60(55 \%)$ & $15 / 60(25 \%)$ & $3 / 60(5 \%)$ & $9 / 60(15 \%)$ \\
\hline & NC-MRA & $6 / 58$ (10\%) & $9 / 58$ (15\%) & $17 / 58(29 \%)$ & $26 / 58(44 \%)$ \\
\hline
\end{tabular}

Abbreviations: ATA, anterior tibial artery; CE-MRA, contrast-enhanced MR angiography; CFA, common femoral artery; CIA, common iliac artery; CPA, common peroneal artery; CTA, CT angiography; EIA, external iliac artery; NC-MRA, noncontrast MR angiography; PTA, posterior tibial artery; SFA, superficial femoral artery.

a(50 segments-aorta; $2 \times \mathrm{CIA} ; 2 \times$ EIA $=5$ segments per patient).

$\mathrm{b}(60$ segments $-2 \times$ CFA; $2 \times$ SFA; $2 \times$ popliteal $)=6$ segments per patient. One artery was not visualized in entirety in the CE-MRA/CTA and NC-MRA and was not included in the grading, hence a total of 59 segments were only analyzed).

' $(60$ segments $-2 \times$ ATA; $2 \times$ PTA; $2 \times$ CPA $)=6$ segments per patient. A total of 60 segments were analyzed in the CE-MRA/CTA. In NC-MRA, 2 arteries were not visualized in entirety and not included in the grading; hence, a total of 58 segments were only analyzed).

MRA was $3.10 \pm 1.13$ and the CE-MRA/CTA was $3.64 \pm 0.77$. The mean scores for each artery individually are as given in - Table 2.

Overall, 77\% (130/169) segments were graded as excellent and $16 \%(27 / 169)$ segments were graded as good in the CE-MRA/CTA, while 53\% (89/167) segments were graded as excellent and 19\% (31/167) segments were graded as good in the NC-MRA. The percentage of segments graded as excellent, good and fair in the aortoiliac, femoropopliteal, and infrapopliteal regions is detailed in - Table 3. As seen in -Table 3, almost 74\% (43/58) of the infrapopliteal arterial segments were graded as fair to poor on the NC-MRA, while $20 \%(12 / 60)$ of these were graded as fair to poor in the CEMRA.

Assessing the Degree of Stenosis

The median degree of stenosis (on a scale from 0 to 3 ) was found to be 1.00 in both NC-MRA and CE-MR/CTA, while the mean was found to be $1.38 \pm 1.31$ and $1.16 \pm 1.26$, respectively (-Table 4).

Of the 170 arterial segments studied, 95 arterial segments were diseased, with 59\% (56/95) segments showing more than $50 \%$ degree of stenosis (significant stenosis) on the

Table 4 Mean and median of the degree of stenosis in all arterial segments

\begin{tabular}{|l|l|l|l|l|}
\hline & & $\begin{array}{c}\text { Contrast } \\
\text { MRA/CTA }\end{array}$ & NC-MRA value \\
\hline Degree of stenosis & $1(0-3)$ & $1(0-3)$ & 0.016 \\
\cline { 2 - 5 } & Median & $1.16 \pm 1.26$ & $1.38 \pm 1.31$ \\
\hline
\end{tabular}

Abbreviations: CE-MRA, contrast-enhanced MR angiography; CTA, CT angiography; NC-MRA, noncontrast MR angiography. 
Table 5 Percentage of arteries which showed significant stenosis (more than $50 \%$ ) region-wise

\begin{tabular}{|l|l|l|}
\hline Arterial region & CE-MRA/CTA & NC-MRA \\
\hline Aortoiliac $^{\text {a }}$ & $13 / 50(26 \%)$ & $18 / 50(36 \%)$ \\
\hline Femoropopliteal $^{\text {b }}$ & $16 / 60(27 \%)$ & $16 / 60(27 \%)$ \\
\hline Infrapopliteal c $^{\text {c }}$ & $27 / 60(45 \%)$ & $41 / 60(68 \%)$ \\
\hline
\end{tabular}

Abbreviations: ATA, anterior tibial artery; CE-MRA, contrast-enhanced MR angiography; CFA, common femoral artery; CIA, common iliac artery; CPA, common peroneal artery; CTA, CT angiography; EIA, external iliac artery; NC-MRA, noncontrast MR angiography; PTA, posterior tibial artery; SFA, superficial femoral artery.

a(50 segments - aorta; $2 \times \mathrm{CIA} ; 2 \times$ EIA $=5$ segments per patient).

${ }^{\mathrm{b}}(60$ segments $-2 \times$ CFA; $2 \times$ SFA $; 2 \times$ popliteal $)=6$ segments per patient).

${ }^{\mathrm{c}}(60$ segments $-2 \times$ ATA; $2 \times$ PTA; $2 \times \mathrm{CPA})=6$ segments per patient $)$.

CE-MRA/CTA, while 79\% (75/95) segments were diagnosed with significant stenosis in the NC-MRA. The number of arteries which had significant stenosis in aortoiliac, femoropopliteal, and infrapopliteal regions is described in - Table 5 .

The longest (craniocaudal) segment measured $19.8 \mathrm{~cm}$ in the NC-MRA and $18.2 \mathrm{~cm}$ in the CE-MRA/CTA, both involving the superficial femoral artery.

The sensitivity, specificity, positive predictive value and negative predictive value of NC-MRA for detecting significant stenosis in the aortoiliac, femoropopliteal, and infrapopliteal region are shown in - Table $\mathbf{6}$. It depicts high sensitivity and specificity in the femoropopliteal region, and a high specificity in the aortoiliac region. On analyzing the NC-MRA and the CE-MRA/CTA using the weighted Cohen's kappa, there was substantial agreement for the aortoiliac region (weighted kappa 0.646 (95\% CI) $(0.361-0.931)(p<0.001)$ (-Table 7). Agreement was almost perfect in the femoropopliteal region with weighted kappa $0.911(95 \% \mathrm{CI})(0.79-1.032)(p<0.001)$. In comparison, for the infrapopliteal region, there was none to slight agreement (weighted kappa 0.052 (95\% CI) $(0.189$ $0.293)(p<0.33587)$, with the NC-MRA mostly overestimating the degree of stenosis.

As compared with the CE-MRA/CTA, the degree of stenosis was overestimated (less than $50 \%$ degree of stenosis on CE-MRA/CTA being labeled as a significant/more than $50 \%$ degree of stenosis on NC-MRA) in two arteries in the aortoiliac region, one artery in the femoropopliteal region, and in 20 arteries in the infrapopliteal region, of which 11 involved the CPA. The NC-MRA underestimated (a
Table 7 Weighted Kappa for the agreement between the two radiologists for the degree of stenosis

\begin{tabular}{|l|l|l|}
\hline Arterial segment & $\begin{array}{l}\text { Weighted Kappa } \\
\text { with 95\% Cl }\end{array}$ & $\begin{array}{l}\text { Observed } \\
\text { agreement }\end{array}$ \\
\hline Aortoiliac & $0.646(0.361,0.931)$ & 0.9 \\
\hline Femoropopliteal & $0.911(0.79,1.032)$ & 0.96667 \\
\hline Infrapopliteal & $0.052(0.189,0.293)$ & 0.516 \\
\hline
\end{tabular}

significant/more than $50 \%$ degree of stenosis on CE-MRA/CTA being labeled as a less than $50 \%$ degree of stenosis on NC-MRA) the degree of stenosis in only 8 arteries (of which 7 were in the infrapopliteal region and the other 1 in the femoropopliteal region). Overall, in 7 out of 10 patients, degree of stenosis was overestimated in the NCMRA, with a majority affecting the infrapopliteal regions.

Concurrence among Radiologists

To assess the reliability of the study, weighted kappa was also calculated to assess the agreement between the degree of stenosis and the quality of images between the two radiologists. Regarding the scoring of image quality, the observed agreement between the two radiologists was 97.52\% (weighted kappa 0.919 (95\% CI) $(0.887,0.952)$ $p<0.001)$. In terms of the grading of the degree of stenosis, the observed agreement between the two radiologists was 97.45\% (weighted kappa 0.945 (95\% CI) (0.923-0.967) $(p<0.001)$.

\section{Discussion}

Over the years, various NC-MRA techniques have been developed in the evaluation of PAD.

TOF MRA, one of the older NC-MRA techniques, a nonsubtractive inflow-dependent technique generates vesselto-background contrast, which is caused by the inflow of fresh, unsaturated blood in a saturated slice of background tissue. ${ }^{12}$ It uses a saturation band on the venous side of the imaging slice and thus aids to null the signal and mask the venous flow. Saturation of the blood flow in the image volume and poor vessel visualization may occur due to retrograde filling, tortuous vessels, slow blood flow or stasis, or vessels which are in the same plane as the image slice. The main disadvantages of TOF include long acquisition time and saturation effect, overestimation of the severity of stenosis, ${ }^{18}$ and increased susceptibility to artifacts. ${ }^{2}$

Table 6 Diagnostic efficacy of NC-MRA as compared with the CE-MRA

\begin{tabular}{|l|l|l|l|l|}
\hline Arterial segment & Sensitivity (\%) & Specificity (\%) & $\begin{array}{l}\text { Positive } \\
\text { predictive } \\
\text { value (\%) }\end{array}$ & $\begin{array}{l}\text { Negative } \\
\text { predictive } \\
\text { value (\%) }\end{array}$ \\
\hline Aortoiliac & 75 & 92.9 & 66.7 & 95.1 \\
\hline Femoropopliteal & 93.3 & 97.8 & 93.3 & 97.8 \\
\hline Infrapopliteal & 58.3 & 47.2 & 42.4 & 63 \\
\hline
\end{tabular}

Abbreviations: CE-MRA, contrast-enhanced MR angiography; NC-MRA, noncontrast MR angiography.

MRA/CTA, depicting high sensitivity and specificity in the femoropopliteal region and a high specificity in the aortoiliac region. 
Some of the further advances in NC-MRA include ECGgated fast spin echo (FSE) MRA, SSFP angiography, flowsensitive dephasing (FSD), inflow-dependent inversion recovery (IFDIR) and arterial spin labeling (ASL) with SSFP technique. $^{19,20}$

SSFP angiography, a GRE-based sequence, has evolved as an improved technique for the imaging of PAD. The advantages of SSFP MRA include short acquisition times and high signal-to-noise ratio ${ }^{21}$ and flow-independent 3D acquisition, with flow compensated in all three directions; its limitations are its susceptibility to field heterogeneities and high-signal intensity in arteries and veins due to background signals. ${ }^{22,23}$

In the beginning of our study, we attempted bSSFP sequence for evaluation of the lower limbs (femoropopliteal and infrapopliteal region). However, the images obtained were extremely suboptimal. The newer techniques like quiescent-interval slice-selective (QISS) are expensive and are not widely available. Thus, we decided to evaluate the efficacy of a combination of these two techniques (bSSFP for the aortoiliac region, and 2D TOF for the femoropopliteal and infrapopliteal regions) in the evaluation of PAD. This might be especially useful in institutions with resource constraints, where newer and expensive techniques of NC-MRA are not available.

Quality of Images

In the aorta, the common femoral, superficial femoral and popliteal arteries, the quality of the images on NC-MRA showed no significant difference from the quality of images of these arteries in the CE-MRA/CTA (-Table 2) (-Figs. 2 and 3 ).

In the aortoiliac region, poorer image quality in the NCMRA as compared with the CE-MRA/CTA was likely due to soft tissue and venous contamination and breathing artifacts. In the infrapopliteal regions, this could probably be attributed to motion artifacts, slower velocities and the smaller caliber of the vessels. Significant PAD with stenosis in the proximal arteries, resulting in poor inflow, may have also been a factor adding to the poor signal in the distal arteries.

Degree of Stenosis

In the aortoiliac and femoropopliteal regions, there was an acceptable agreement between the NC-MRA and the
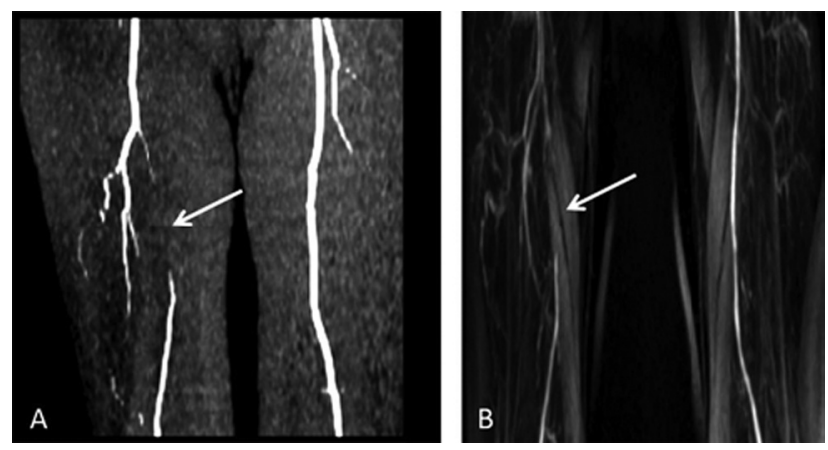

Fig. 3 Noncontrast MR angiography (NC-MRA) and contrast-enhanced MR angiography (CE-MRA) in the same patient in the femoropopliteal region. (A) NC-MRA: grade 3 stenosis in the right superficial femoral artery (white arrow). (B) CE-MRA: grade 3 stenosis in the right superficial femoral artery (white arrow).
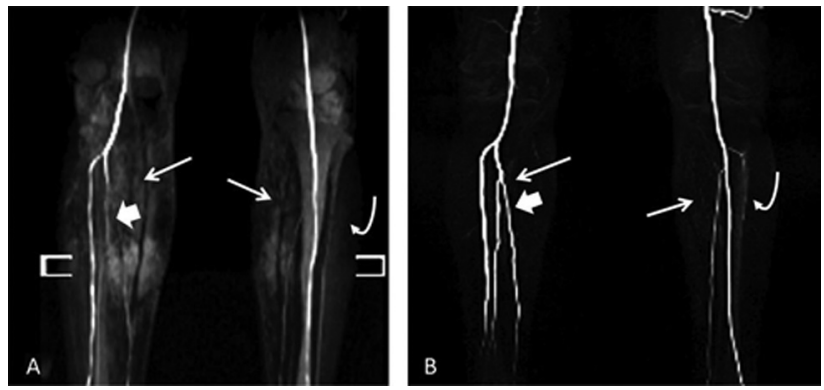

Fig. 4 Noncontrast MR angiography (NC-MRA) and contrast-enhanced MR angiography (CE-MRA) in the same patient in the infrapopliteal region. (A) NC-MRA of the infrapopliteal arteries show grade 3 stenosis of the right posterior tibial artery (PTA) (white arrow) and anterior tibial artery (ATA) proximally (block white arrow) the left PTA (white arrow) and the left common peroneal artery (CPA) (curved white arrow). (B) CE-MRA of the infra-popliteal arteries shows that the right PTA (white arrow) and proximal right ATA (block white arrow) are patent with grade 2 stenosis of the left PTA (white arrow) and grade 3 stenosis of the left CPA (curved white arrow).

CE-MRA/CTA. But in the infrapopliteal region, the agreement was poor. The NC-MRA overestimated the degree of stenosis (-Fig. 4), more so in the infrapopliteal arteries, with the CPA being the most affected. This could probably be attributed to the smaller caliber of the arteries in the leg and poor inflow due to proximal stenosis. The length of the stenosis was also overestimated by the NC-MRA, especially when the length of the stenosis was relatively shorter. The quality of NC-MRA in the aortoiliac region was good on visual interpretation. However, there was overestimation of stenosis in the aortoiliac region, which is likely due to decrease in flow velocity in the poststenotic vascular segments. ${ }^{24,25}$ Overestimation of the stenosis in the femoropopliteal and infrapopliteal regions is likely due to the flow-dependent nature of the TOF technique.

Although we attempted to reduce motion by alleviating pain by administering oral analgesics prior to the study, motion-related artifacts could have contributed to the poorer performance of the NC-MRA, which takes more time than CE-MRA (19 minutes vs. 4 minutes).

In the evaluation of the arteries of the femoropopliteal region and the infrapopliteal region, the results of our study were comparable to that by Suttmeyer et al. They found a good correlation between the NC-MRA (using 2D TOF) and DSA, with a poor correlation in the infrapopliteal arteries in an open 1.0 Tesla MRI. ${ }^{26}$ Gozzi et al analyzed the arteries from the celiac trunk to the feet by comparing unenhanced TOF sequences to DSA in the evaluation of PAD in 30 patients. They found a sensitivity of $96 \%$ and sensitivity of $99 \%$ of unenhanced TOF as compared with DSA, with a high sensitivity and specificity even in the arteries of the calf and feet. ${ }^{27}$ As compared with our study, they utilized a smaller flip angle, a smaller field of view (FOV), a larger matrix, and a head coil while assessing the arteries of the feet. Superficial coils may have aided in further enhancing the quality of the images in the infrapopliteal region; however, this was not utilized in our study. In a meta-analysis by Nelemans et al, 
the relative odds ratio of CE-MRA with NC-MRA using 2D TOF angiography was found to be 7.46 (95\% CI: $2.48,22.20){ }^{28}$

In the evaluation of abdominal vessels using bSSFP, the specificity and negative predictive value of our study was similar to a study by Cardia et al, who evaluated the stenosis of the celiac trunk and the superior mesenteric artery using bSSFP. ${ }^{24}$ Studies by Maki et al and Wyttenbach et al also showed similar specificity of SSFP in the evaluation of renal artery stenosis. ${ }^{29,30}$ Lanzman et al found similar results in the evaluation of transplant renal artery. ${ }^{31}$ As seen in our study, Caria et al also found NC-MRA to overestimate the stenosis in arterial sgements. ${ }^{24}$ Lanzman et al also demonstrated that SSFP using 3T significantly improved the visualization of renal arteries as compared with a $1.5 \mathrm{~T} \mathrm{MRI.}{ }^{32}$

Single-shot radial QISS, another inflow-dependent nonsubtractive technique for NC-MRA, is ECG-gated and uses a bSSFP readout. QISS is deemed to have certain advantages over other types of noncontrast MRA, with significantly reduced scan times (thus reducing motion-related artifacts) and allows a large number of slices to be acquired in a single breath hold. ${ }^{33-37}$ As compared with DSA and CE-MRA, QISS has demonstrated a high sensitivity and specificity in the evaluation of PAD, ${ }^{34-36}$ with a median sensitivity and specificity of $91.4 \%$ and $96.4 \%$ when compared with DSA and a median sensitivity and specificity of $89.2 \%$ and $96.0 \%$ when compared with CE-MRA. ${ }^{37}$

Limitations of the Study

The study group comprised a small number of patients. However, the analysis was performed on the individual arterial segments amounting to 170 arterial segments. Another limitation of the study is that DSA was not used as the gold standard, as it is an invasive procedure. In the infrapopliteal region, the quality of the images in the CE-MRA/CTA was graded as poor in approximately $15 \%$ of the arteries. Arterial segments, which were not visualized, could not be included in the analysis of image quality, as we could not determine whether this was due to occlusion or poor image quality. This could have resulted in spuriously better grading of image quality.

\section{Conclusion}

In conclusion, NC-MRA, using a combination of bSSFP technique for aortoiliac vessels and 2D inflow dependent TOF technique for the lower limb arteries, was found to be comparable to CE-MRA/CTA in the evaluation of lower limb PAD in the aortoiliac and femoropopliteal regions. The quality of images and ability to detect and quantify stenoses fared well in assessing the aortoiliac vessels and femoropopliteal vessels, with suboptimal imaging of infrapopliteal arteries. In resource limited centers, where newer software may not be available, a combination of these NCMRA techniques as described in our study may serve to optimally evaluate patients with PAD and facilitate and plan further patient management like endovascular intervention or surgery. Research on further refinements of the NC-MRA techniques for better evaluation of the infrapopliteal arteries continue to open more avenues for the evaluation of PAD.

\section{Funding}

Nil.

a. pvalue $>0.05$ in the aorta, the common femoral, superficial femoral and popliteal arteries, implying that the mean of the scores of the quality in these segments in the NCMRA and CE-MRA/CTA were statistically not different from each other

b. pvalue of 0.05 taken as statistically significant. Low $p$ values in the infrapopliteal arteries indicate statistically significant better image quality score on CE-MRA as compared with NCMRA.

\section{Conflicts of Interest}

None declared.

\section{Acknowledgments}

The authors would like to thank the Department of Vascular Surgery, Christian Medical College, Vellore, India. The authors would also like to thank Mr. Victor Savariappan and Mr. Walter Nixon, senior radiographers in the Department of Radiology, Christian Medical College, Vellore, India, for technical assistance and Dr. Pippa Deodhar for editing the manuscript.

\section{References}

1 Al-Rudaini HEA, Han P, Liang H. Comparison between computed tomography angiography and digital subtraction angiography in critical lower limb ischemia. Curr Med Imaging Rev 2019;15(05): 496-503

2 Edelman RR, Koktzoglou I. Noncontrast MR angiography: an update. J Magn Reson Imaging 2019;49(02):355-373

3 Hirsch AT, Haskal ZJ, Hertzer NRet al.American Association for Vascular Surgery; Society for Vascular Surgery; Society for Cardiovascular Angiography and Interventions; Society for Vascular Medicine and Biology; Society of Interventional Radiology; ACC/ AHA Task Force on Practice Guidelines Writing Committee to Develop Guidelines for the Management of Patients With Peripheral Arterial Disease; American Association of Cardiovascular and Pulmonary Rehabilitation; National Heart, Lung, and Blood Institute; Society for Vascular Nursing; TransAtlantic Inter-Society Consensus; Vascular Disease Foundation. ACC/AHA 2005 Practice Guidelines for the management of patients with peripheral arterial disease (lower extremity, renal, mesenteric, and abdominal aortic): a collaborative report from the American Association for Vascular Surgery/Society for Vascular Surgery, Society for Cardiovascular Angiography and Interventions, Society for Vascular Medicine and Biology, Society of Interventional Radiology, and the ACC/AHA Task Force on Practice Guidelines (Writing Committee to Develop Guidelines for the Management of Patients With Peripheral Arterial Disease): endorsed by the American Association of Cardiovascular and Pulmonary Rehabilitation; National Heart, Lung, and Blood Institute; Society for Vascular Nursing; TransAtlantic Inter-Society Consensus; and Vascular Disease Foundation. Circulation 2006;113(11):e463-e654

4 Thierfelder KM, Meimarakis G, Nikolaou Ket al.Non-contrastenhanced MR angiography at 3 Tesla in patients with advanced peripheral arterial occlusive disease. PLoS One 2014;9(03): e91078

5 Ersoy H, Rybicki FJ. MR angiography of the lower extremities. AJR Am J Roentgenol 2008;190(06):1675-1684

6 Aboyans V, Ricco J-B, Bartelink MEL, et al.ESC Scientific Document Group. 2017 ESC Guidelines on the Diagnosis and Treatment of Peripheral Arterial Diseases, in collaboration with the European 
Society for Vascular Surgery (ESVS): Document covering atherosclerotic disease of extracranial carotid and vertebral, mesenteric, renal, upper and lower extremity arteriesEndorsed by: the European Stroke Organization (ESO)The Task Force for the Diagnosis and Treatment of Peripheral Arterial Diseases of the European Society of Cardiology (ESC) and of the European Society for Vascular Surgery (ESVS). Eur Heart J 2018;39(09):763-816

7 Gerhard-Herman MD, Gornik HL, Barrett Cet al.2016 AHA/ACC Guideline on the Management of Patients With Lower Extremity Peripheral Artery Disease: Executive Summary: A Report of the American College of Cardiology/American Heart Association Task Force on Clinical Practice Guidelines. J Am Coll Cardiol 2017;69 (11):1465-1508

8 Costello JR, Kalb B, Martin DR. Incidence and risk factors for gadolinium-based contrast agent immediate reactions. Top Magn Reson Imaging 2016;25(06):257-263

9 Agarwal R, Brunelli SM, Williams K, Mitchell MD, Feldman HI, Umscheid CA. Gadolinium-based contrast agents and nephrogenic systemic fibrosis: a systematic review and meta-analysis. Nephrol Dial Transplant 2009;24(03):856-863

10 Leisman S. Radiocontrast toxicity. Adv Chronic Kidney Dis 2020; 27(01):50-55

11 Woolen SA, Shankar PR, Gagnier JJ, MacEachern MP, Singer L, Davenport MS. Risk of nephrogenic systemic fibrosis in patients with stage 4 or 5 chronic kidney disease receiving a group ii gadolinium-based contrast agent: a systematic review and metaanalysis. JAMA Intern Med 2020;180(02):223-230

12 Leiner T. Magnetic resonance angiography of abdominal and lower extremity vasculature. Top Magn Reson Imaging 2005;16 (01):21-66

13 François CJ, Tuite D, Deshpande V, Jerecic R, Weale P, Carr JC. Unenhanced MR angiography of the thoracic aorta: initial clinical evaluation. AJR Am J Roentgenol 2008;190(04):902-906

14 Van Grimberge F, Dymarkowski S, Budts W, Bogaert J. Role of magnetic resonance in the diagnosis of subclavian steal syndrome. J Magn Reson Imaging 2000;12(02):339-342

15 Miyazaki M, Sugiura S, Tateishi F, Wada H, Kassai Y, Abe H. Noncontrast-enhanced MR angiography using 3D ECG-synchronized half-Fourier fast spin echo. J Magn Reson Imaging 2000;12(05): 776-783

16 McHugh ML. Interrater reliability: the kappa statistic. Biochem Med (Zagreb) 2012;22(03):276-282

17 Viera AJ, Garrett JM. Understanding interobserver agreement: the kappa statistic. Fam Med 2005;37(05):360-363

18 U-King-Im JM, Young V, Gillard JH. Carotid-artery imaging in the diagnosis and management of patients at risk of stroke. Lancet Neurol 2009;8(06):569-580

19 Mathew RC, Kramer CM. Recent advances in magnetic resonance imaging for peripheral artery disease. Vasc Med 2018;23(02): 143-152

20 Morita S, Masukawa A, Suzuki K, Hirata M, Kojima S, Ueno E. Unenhanced MR angiography: techniques and clinical applications in patients with chronic kidney disease. Radiographics 2011;31(02):E13-E33

21 Foo TKF, Ho VB, Marcos HB, Hood MN, Choyke PL. MR angiography using steady-state free precession. Magn Reson Med 2002;48(04): 699-706

22 Katoh M, Spuentrup E, Stuber M, Hoogeveen R, Günther RW, Buecker A. Free-breathing renal magnetic resonance angiography with steady-state free-precession and slab-selective spin inversion combined with radial k-space sampling and water-selective excitation. Magn Reson Med 2005;53(05):1228-1233

23 Scheffler K, Lehnhardt S. Principles and applications of balanced SSFP techniques. Eur Radiol 2003;13(11):2409-2418

24 Cardia PP, Penachim TJ, Prando A, Torres US, D'Ippólito G. Noncontrast MR angiography using three-dimensional balanced steady-state free-precession imaging for evaluation of stenosis in the celiac trunk and superior mesenteric artery: a preliminary comparative study with computed tomography angiography. $\mathrm{Br} \mathrm{J}$ Radiol 2017;90(10/75):20170011

25 Perry JT, Statler JD. Advances in vascular imaging. Surg Clin North Am 2007;87(05):975-993, vii

26 Suttmeyer B, Teichgräber U, Thomas Aet al.Non-invasive ECGtriggered 2D TOF MR angiography of the pelvic and leg arteries in an open 1.0-tesla high-field MRI system in comparison to conventional DSA. Biomed Tech (Berl) 2014;59(01):29-37

27 Gozzi M, Amorico MG, Colopi Set al.Peripheral arterial occlusive disease: role of MR angiography. Radiol Med (Torino) 2006;111 (02):225-237

28 Nelemans PJ, Leiner T, de Vet HCW, van Engelshoven JMA. Peripheral arterial disease: meta-analysis of the diagnostic performance of MR angiography. Radiology 2000;217(01): 105-114

29 Maki JH, Wilson GJ, Eubank WB, Glickerman DJ, Millan JA, Hoogeveen RM. Navigator-gated MR angiography of the renal arteries: a potential screening tool for renal artery stenosis. AJR Am J Roentgenol 2007;188(06):W540-6

30 Wyttenbach R, Braghetti A, Wyss Met al.Renal artery assessment with nonenhanced steady-state free precession versus contrastenhanced MR angiography. Radiology 2007;245(01):186-195

31 Lanzman RS, Voiculescu A, Walther Cet al.ECG-gated nonenhanced 3D steady-state free precession MR angiography in assessment of transplant renal arteries: comparison with DSA. Radiology 2009;252(03):914-921

32 Lanzman RS, Kröpil P, Schmitt Pet al.Nonenhanced free-breathing ECG-gated steady-state free precession 3D MR angiography of the renal arteries: comparison between $1.5 \mathrm{~T}$ and $3 \mathrm{~T}$. AJR Am J Roentgenol 2010;194(03):794-798

33 Edelman RR, Silvers RI, Thakrar KHet al.Nonenhanced MR angiography of the pulmonary arteries using single-shot radial quiescent-interval slice-selective (QISS): a technical feasibility study. J Cardiovasc Magn Reson 2017;19(01):48

34 Hanrahan CJ, Lindley MD, Mueller Met al.Diagnostic accuracy of noncontrast $\mathrm{mr}$ angiography protocols at $3 \mathrm{~T}$ for the detection and characterization of lower extremity peripheral arterial disease. J Vasc Interv Radiol 2018;29(11):1585-1594.e2

35 Varga-Szemes A, Wichmann JL, Schoepf UJet al.Accuracy of noncontrast quiescent-interval single-shot lower extremity $\mathrm{mr}$ angiography versus ct angiography for diagnosis of peripheral artery disease. JACC Cardiovasc Imaging 2017;10(10):1116-1124

$36 \mathrm{Wu} \mathrm{G}$, Yang J, Zhang Tet al.The diagnostic value of non-contrast enhanced quiescent interval single shot (QISS) magnetic resonance angiography at 3T for lower extremity peripheral arterial disease, in comparison to CT angiography. J Cardiovasc Magn Reson 2016;18(01):71

37 Cavallo AU, Koktzoglou I, Edelman RRet al.Noncontrast magnetic resonance angiography for the diagnosis of peripheral vascular disease. Circ Cardiovasc Imaging 2019;12(05):e008844 